\title{
Comparison of the diagnostic efficacy between ultrasound elastography and magnetic resonance imaging for breast masses
}

\author{
RONG CHENG, JING LI, LI JI, HUINING LIU and LIMIN ZHU
}

Department of Ultrasound, Nuclear Industry 215 Hospital of Shanxi Province, Xianyang, Shanxi 712000, P.R. China

Received March 23, 2016; Accepted March 3, 2017

DOI: $10.3892 / \mathrm{etm} .2017 .5674$

\begin{abstract}
The present study compared the efficacy of ultrasound elastography (UE), magnetic resonance imaging (MRI) and the combination of the two methods (UE+MRI) in the differential diagnosis of benign and malignant breast tumors. In total, 86 patients with breast masses were recruited and evaluated by UE, MRI and UE+MRI. Strain ratios of UE were calculated for the breast mass and adjacent normal tissues. In addition, the receiver operating characteristic (ROC) curve was obtained, while the sensitivity and specificity were calculated to determine the optimal cut-off point for the differential diagnosis. The area under the ROC curve (AUC) was also calculated to evaluate the diagnostic performance of these methods. The results indicated that the diagnostic accuracy of UE+MRI was significantly higher compared with the UE or MRI methods in the differential diagnosis of invasive ductal, invasive lobular, intraductal papillary, medullary and mucinous carcinomas (all $\mathrm{P}<0.05$ ). The optimal cut-off points of ROC curve of the Strain Ratio in the diagnosis of breast lesions were 2.81, 3.76 and 3.42 for UE, MRI and UE+MRI, respectively. Furthermore, the AUC values were 86.7, 79.2 and 91.4\%, while the diagnostic accuracy rates were 82.5, 75.5 and 95.3\%, for UE, MRI and UE+MRI, respectively. Accuracy rate differences between UE and MRI or between UE and UE+MRI were statistically significant $(\mathrm{P}<0.05)$, whereas no significant difference existed between MRI and UE+MRI (P>0.05). Finally, the diagnostic consistency of the UE+MRI method with the pathological diagnosis was higher compared with UE or MRI alone. In conclusion, the combination of UE and MRI is superior to the use of UE or MRI alone in the differential diagnosis of benign and malignant breast masses.
\end{abstract}

Correspondence to: Dr Jing Li, Department of Ultrasound, Nuclear Industry 215 Hospital of Shanxi Province, 35 Weiyang Road, Xianyang, Shanxi 712000, P.R. China

E-mail: lijing0090@126.com

Key words: diagnostic efficacy, ultrasound elastography, strain ratio, magnetic resonance imaging, breast mass

\section{Introduction}

A solid breast mass, with a wide variation in the radiological appearance corresponding to different properties of the pathological features, can be broadly classified as benign or malignant (1). Thus, differential diagnosis of solid breast masses is of great significance in clinical therapy and prognostic evaluation. Breast cancer is one of the most common malignant solid tumors in women, occurring in $\sim 1.5$ million women worldwide each year (2). The incidence of breast cancer is rising each year and patients tend to be affected at a younger age $(3,4)$. Along with the improvement of imaging diagnostic technology for breast diseases in recent years, early diagnosis and treatment have been implemented, which significantly improved the 5-year survival rate and life quality of patients with breast cancer $(5,6)$. Therefore, early and sensitive diagnostic methods result in therapy at an earlier stage of the disease and a better prognosis. Previous studies have demonstrated that the 10 -year survival rate was up to $80 \%$ in patients with breast cancer when early and suitable treatment was administrated $(7,8)$.

Currently, there are various imaging methods for non-invasive diagnosis of breast masses, including conventional ultrasonography, X-ray mammography, multi-slice spiral computed tomography, magnetic resonance imaging (MRI) and Doppler ultrasound color flow imaging. However, each single method has various advantages and disadvantages, and the results obtained from different methods are often conflicting. Therefore, the combination of two or three diagnostic methods is commonly adopted in determining the properties of breast masses and for the clinical diagnosis of breast cancer $(9,10)$.

$\mathrm{X}$-ray mammography is the most important tool in the screening of breast cancer in clinical practice. However, MRI has higher sensitivity than X-ray mammography in identifying occult breast lesions, particularly dense lesions (11). MRI can provide a more reliable basis for accurate staging and the development of a clinical treatment strategy for breast cancer. Ultrasound elastography (UE) is another examination technology for breast cancer with a rapid development in recent years. UE is a method used to visualize the elasticity of tumors and, thus, demonstrates more superior advantages in differentiating benign and malignant breast tumors compared with conventional ultrasonography (12). The strain ratio (SR) and a five-point scoring system are the two most useful and 
effective parameters to consider in UE. SR-based elastographic analysis can provide a novel and more reliable diagnostic tool in comparison to a five-point scoring system for UE in the diagnosis of breast cancer (13).

The aim of the present study was to evaluate and compare the performance of UE, MRI and the combination of these two methods (UE+MRI) in the diagnosis of breast cancer and its differentiation from benign lesions.

\section{Materials and methods}

Patient recruitment. Between October 2014 and October 2015, a total of 86 patients with solid lesions in the breast who were admitted to the Department of Breast Surgery of the Nuclear Industry 215 Hospital of Shanxi Province (Xianyang, China) were included in the study. All patients underwent diagnostic examination with UE and MRI. The patients were randomly divided into the UE+MRI $(n=43)$, UE $(n=26)$ and MRI $(n=17)$ groups. The inclusion criteria were as follows: i) Patients had newly diagnosed breast nodules; ii) the breast lesions were solid or mixed (cystic-solid) nodules, with or without calcification; iii) patients voluntary underwent the UE or MRI examination; and iv) patients signed informed consent prior to surgery or biopsy. The exclusion criteria were as follows: i) Patients with cystic nodules and ii) patients with malignant nodules. The clinical characteristics of patients and histological features of the breast lesions are shown in Table I. Prior written and informed consent was obtained from every patient, and the study was approved by the Ethics Review Board of the Nuclear Industry 215 Hospital of Shanxi Province.

UE examination. UE was used to analyze the properties of breast masses. UE examination was performed using Philips IU Elite color Doppler ultrasound system (Philips Medical Systems, Bothell, WA, USA) equipped with UE capacity and a L11-3 linear array transducer. Firstly, two-dimensional ultrasound scanning was performed on the patients' breasts to observe the number, location and size of the masses. Real-time UE analysis was then conducted at the lesion site. The blue, green and red images represented the strong tissue stiffness, moderate tissue stiffness and soft tissue, respectively. Next, the elastographic imaging was adjusted to an appropriate size corresponding to the lesion size. The linear probe was maintained at the lesion site and a slight vibration (compression/decompression operation) was performed. Two-dimensional and elasticity images were observed on a real-time display. The region of interest (ROI) was set for the lesion tissue and surrounding normal breast tissue in the same depth as the breast lesion. Finally, the SR value was calculated to assess the relative hardness of the breast lesion vs. the surrounding breast tissue. Examination for each lesion was conducted in triplicate and the average SR value was calculated.

MRI examination. All patients were placed in prone position and examined on a 1.5-Tesla MRI scanner equipped with a dedicated breast surface coil (Magnetom Avanto; Siemens Medical Solutions, Erlangen, Germany). The scanning range included the bilateral breasts and the corresponding level of prothoraxes and bilateral axillae. The breast MRI protocol included axial T1-weighted FLASH, T2-weighted TIRM and diffusion-weighted single-shot echo-planar MRI sequences, as well as short inversion time inversion recovery as a technique of fat suppression. The pathological features of benign and malignant breast tumors in the MRI scans were as follows: Benign tumors were generally round or oval in shape with a clear boundary or lobulated appearance, whereas the edges of malignant tumors were blurry and displayed irregular with unclear boundaries from the surrounding tissues. Finally, the differential diagnosis of benign and malignant breast masses was performed using the Breast Imaging Reporting and Data System recommended by the American College of Radiology (14). The likelihood of malignancy of each lesion was classified according to a five-point scale (15) as follows: 1 , benign lesion; 2 , potentially benign; 3 , possibly benign but in need of follow-up; 4, potentially malignant; and 5, malignant tumor. Generally, tumors classified on the scale as 1-3 were considered benign, while those classified as 4 and 5 were considered malignant tumors. Classifying scale 3 tumors as benign did not affect the study. Scale 3 refers to tumors that have $98 \%$ chance of benign, but still in need of follow-up. In the present study, the cases of scale 3 were all benign cases, as confirmed by the follow-up data.

Hematoxylin and eosin $(H \& E)$ staining. Pathological results from $H \& E$ staining were used as the golden standard for definitive diagnosis of the breast lesions. Breast tissues were collected from resected breast masses subsequent to surgery, fixed in $10 \%$ formaldehyde and embedded in paraffin. The tissues were cut into $5-\mu \mathrm{m}$ sections and stained with H\&E. The diagnosis of benign or malignant breast tumor was confirmed independently by two pathologists.

Observational parameters and image analysis. Imaging findings in the two groups were evaluated for breast masses including breast fibroadenoma, mastitis, invasive ductal carcinoma, invasive lobular carcinoma, intraductal papillary carcinoma, medullary carcinoma and mucinous carcinoma. The diagnostic performances were compared among the UE, MRI and UE+MRI methods according to their correlation with the pathological diagnosis. In order to determine the optimal cut-off points for the diagnosis of benign and malignant breast masses, a receiver operating characteristic (ROC) curve was constructed. Subsequently, the area under the ROC curve (AUC) was calculated to evaluate the diagnostic performance of these methods, while the detection rate, sensitivity and specificity of the three methods were also determined.

Statistical analysis. All data were analyzed using the SPSS version 17.0 software (SPSS, Inc., Chicago, IL, USA). Comparison of continuous data between two groups was performed by independent-samples t-test, and comparison of categorical data was conducted by $\chi^{2}$ test. Kappa coefficients were calculated. $\mathrm{P}<0.05$ was considered to demonstrated differences that were statistically significant.

\section{Results}

Clinical characteristics of patients and imaging findings. A total of 86 patients with single solid lesions in the breast were included in the present study. UE and MRI were used 
Table I. Clinical characteristics of patients included in the present study.

\begin{tabular}{|c|c|c|c|c|}
\hline Characteristics & UE+MRI group $(n=43)$ & UE group $(n=26)$ & MRI group $(n=17)$ & P-value \\
\hline Age $(\text { years })^{\mathrm{a}}$ & $34.4 \pm 8.7$ & $39.6 \pm 10.6$ & $41.3 \pm 9.7$ & 0.083 \\
\hline Mass location (left/right) & $23 / 20$ & $10 / 16$ & $5 / 12$ & 0.056 \\
\hline Maximum diameter of mass $(0.3-4.0 \mathrm{~cm})^{\mathrm{a}}$ & $2.6 \pm 1.2$ & $2.0 \pm 0.65$ & $2.3 \pm 318$ & 0.094 \\
\hline Axillary lymph nodes and breast palpation (yes/no) & $35 / 8$ & $19 / 7$ & $12 / 5$ & 0.042 \\
\hline Calcification (yes/no) & $24 / 21$ & $11 / 15$ & $6 / 11$ & 0.037 \\
\hline Mass appearance (regular/irregular) & $28 / 15$ & $17 / 9$ & $10 / 7$ & 0.058 \\
\hline Echo intensity of UE (strong/moderate) & $21 / 22$ & $18 / 8$ & $10 / 7$ & 0.023 \\
\hline
\end{tabular}

${ }^{\mathrm{a}}$ Mean \pm standard deviation. UE, ultrasound elastography.

Table II. Comparison of detection rates of UE, MRI and UE+MRI methods.

\begin{tabular}{lccc}
\hline Parameter & UE & MRI & UE+MRI \\
\hline $\begin{array}{l}\text { Definitive diagnosis } \\
\text { Breast fibroma }\end{array}$ & $4(9.3)$ & $3(6.9)$ & $5(11.6)$ \\
Breast hyperplasia & $5(11.6)$ & $2(4.6)$ & $4(9.3)$ \\
Invasive ductal carcinoma & $10(23.2)$ & $9(20.9)$ & $11(25.5)$ \\
Intraductal papillary & $7(16.2)$ & $5(11.6)$ & $7(16.2)$ \\
carcinoma & & & \\
Invasive lobular carcinoma & $8(18.6)$ & $6(13.9)$ & $9(20.9)$ \\
Mucinous carcinoma & $1(2.3)$ & $2(4.6)$ & $3(6.9)$ \\
Medullary carcinoma & $2(4.6)$ & $1(2.3)$ & $1(2.3)$ \\
Detection rate $(\%)$ & 85.8 & 64.8 & 92.7 \\
\hline
\end{tabular}

UE, ultrasound elastography; MRI, magnetic resonance imaging.

to observe the breast masses, which were subsequently evaluated. In the UE+MRI group, patients (mean age, $34.4 \pm 8.7$ years; age range, $27-65$ years) were examined by a combination of UE and MRI methods. The mass was located in the left breast in 23 cases and in the right breast in 20 cases. In addition, the mean maximum diameter of the mass in the UE+MRI group was $2.6 \pm 1.2 \mathrm{~cm}$, with a range of $0.3-4.0 \mathrm{~cm}$. In the UE group (mean age, $39.6 \pm 10.6$ years; age range, 22-67 years) and MRI group (mean age, $41.3 \pm 9.7$ years; age range, 22-70 years), patients were examined by UE or MRI, respectively. The left/right breast mass location ratio was $10 / 16$ in the UE group and 5/12 in the MRI group. The mean maximum diameters of the masses were 2.0 0.5 and $2.3 \pm 0.8 \mathrm{~cm}$ in the UE and MRI groups, respectively (Table I). The benign masses were generally displayed as round or oval lesions with a clear boundary. By contrast, the malignant tumors were blurry, and displayed irregular lesion edges, non-uniform imaging signals and unclear boundaries from the surrounding tissues. Representative UE scans of patients with malignant and benign tumors are shown in Fig. 1A and B, respectively, while MRI scans are shown in Fig. 1C and D. These diagnoses were later confirmed by H\&E staining of tissue samples (Fig. 1E and F).

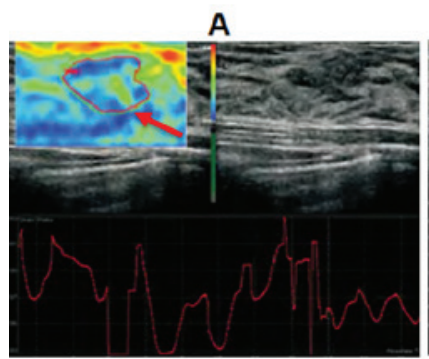

C

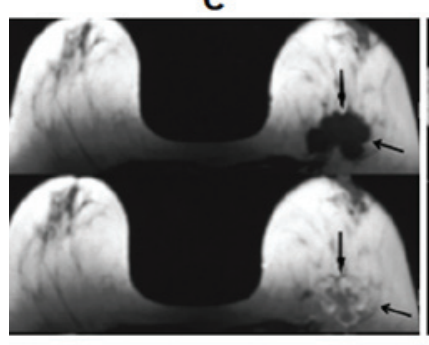

E

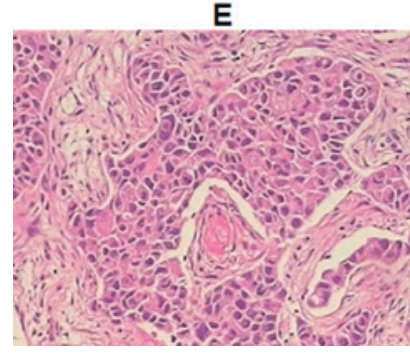

Figure 1. Imaging findings (UE and MRI) and pathological features (H\&E staining) of benign and malignant breast masses. UE imaging of (A) invasive ductal carcinoma and (B) breast fibroma (arrow, region of interest). MRI scans of (C) breast invasive ductal carcinoma (left side; arrows, breast lesion) and (D) breast fibrosis (left side; arrows, breast lesion). $\mathrm{H} \& \mathrm{E}$ staining showing the pathological features of (E) invasive ductal carcinoma (magnification, $\mathrm{x} 40$ ) and $(\mathrm{F})$ breast fibroma (magnification, $\mathrm{x} 40$ ). UE, ultrasound elastography; MRI, magnetic resonance imaging; H\&E, hematoxylin and eosin.

Comparison of detection rate of different methods. The detection rates of the UE, MRI and UE+MRI methods were evaluated based on the pathological analysis. As shown in Table II, there were 28 cases of malignant masses and 9 cases of benign masses in the UE group, 23 malignant masses and 5 benign masses in the MRI group, and 31 malignant masses and 9 benign masses in the UE+MRI group. The detection 

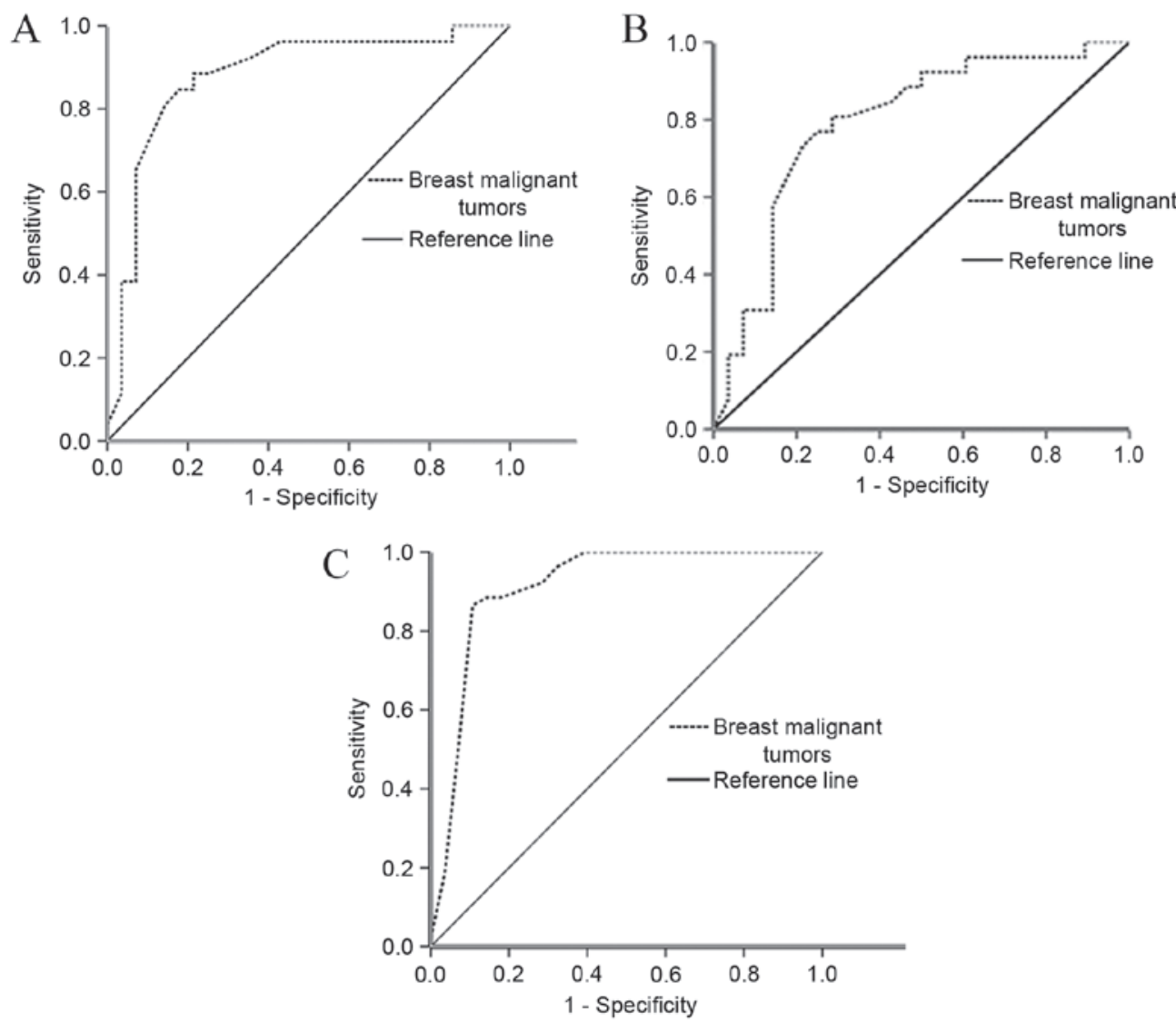

Figure 2. ROC curve with reference line for methods (A) UE, (B) MRI and (C) UE+MRI. The ROC curve was constructed to determine the optimal cut-off points for the diagnosis of benign and malignant breast masses, and to evaluate the diagnostic performance of these methods. ROC, receiver operating characteristic; UE, ultrasound elastography; MRI, magnetic resonance imaging.

rates were $85.8,64.8$ and $92.7 \%$ in the UE, MRI and UE+MRI groups, respectively. The difference in the detection rates between the UE and UE+MRI groups or between the MRI and UE+MRI groups were statistically significant (all $\mathrm{P}<0.05$ ), indicating that the combination of UE and MRI method is superior to the application of UE or MRI alone in the diagnosis of benign and malignant breast masses.

Definition of optimal cut-off points. The ROC curve, with sensitivity as the $\mathrm{y}$-axis and 1-specificity as the $\mathrm{x}$-axis, was constructed to determine the optimal cut-off points for the three methods. The results demonstrated that the cut-off points of UE, MRI and UE+MRI methods were 2.81, 3.76 and 3.42, respectively, in the diagnosis of benign or malignant breast masses (Fig. 2), while the AUC values were 86.7, 79.2 and $91.4 \%$, respectively. The differences in the AUC value between the UE and UE+MRI groups or between the MRI and UE+MRI groups were statistically significant (all $\mathrm{P}<0.05$ ), which also revealed that combination of the UE and MRI diagnostic methods yielded the best results for detection of breast cancer.

Comparison of diagnostic accuracy rates of different methods. A total of 72 cases of malignant breast cancer in the 86 patients were pathologically diagnosed following surgery. For the UE, MRI and UE+MRI diagnostic methods, the following values were observed: The sensitivity values were
83.3, 77.7 and $95.8 \%$; the specificity values were $78.5,64.2$ and $92.8 \%$; and the accuracy rates of differential diagnosis were $82.5,75.5$ and $95.3 \%$, respectively (Table III). The differences in accuracy rates between the UE and MRI methods or between the UE and UE+MRI methods were found to be statistically significant (all $\mathrm{P}<0.05$ ); however, there was no significant difference in the accuracy rate between the MRI and UE+MRI methods ( $P>0.05)$. The Youden index in UE, MRI, and UE+MRI was 61.8, 41.9, and 88.6, respectively. There were significant differences in Youden index among the three methods (all $\mathrm{P}<0.05$; Table III). This suggests that the UE+MRI method has higher test authenticity compared with UE or MRI alone.

Diagnostic consistency of imaging methods with pathological analysis. A total of 72 cases of malignant breast cancer were selected from the 86 patients. These 72 patients were examined by pathology and all three methods. The pathological results were used as the golden standard. A curve-fitting analysis was performed to evaluate the diagnostic consistency of the UE, MRI or UE+MRI findings with the results of pathological analysis. The kappa coefficients for UE, MRI and UE+MRI were $0.512,0.527$ and 0.630 , respectively (all $\mathrm{P}<0.001$ ). These values indicate that the diagnostic consistency of the combination of UE and MRI was higher compared with the application of UE or MRI alone in the diagnosis of benign and malignant breast masses (Table IV). 
Table III. Comparison of diagnostic accuracy rates of UE, MRI and UE+MRI methods.

\begin{tabular}{lccccc}
\hline Group & Sensitivity $(\%)$ & Specificity $(\%)$ & Youden index & Accuracy rate $(\%)$ & P-value \\
\hline UE & 83.3 & 78.5 & 61.8 & 82.5 & 0.023 \\
MRI & 77.7 & 64.2 & 41.9 & 75.5 & 0.057 \\
UE+MRI & 95.8 & 92.8 & 88.6 & 95.3 & 0.006 \\
\hline
\end{tabular}

UE, ultrasound elastography; MRI, magnetic resonance imaging.

Table IV. Diagnostic consistency of the three imaging methods with the pathological diagnosis.

\begin{tabular}{|c|c|c|c|c|c|c|c|}
\hline \multirow[b]{2}{*}{ Group } & \multirow[b]{2}{*}{ Imaging diagnosis } & \multicolumn{2}{|c|}{ Pathological diagnosis } & \multirow[b]{2}{*}{ Total } & \multirow[b]{2}{*}{ Pearson $\chi^{2}$} & \multirow[b]{2}{*}{ Kappa coefficients } & \multirow[b]{2}{*}{ P-value } \\
\hline & & + & - & & & & \\
\hline \multirow[t]{2}{*}{ UE } & + & 60 & 2 & 62 & 47.489 & 0.512 & 0.001 \\
\hline & - & 4 & 6 & 10 & & & \\
\hline \multirow[t]{2}{*}{ MRI } & + & 56 & 7 & 63 & 53.580 & 0.527 & 0.001 \\
\hline & - & 3 & 6 & 9 & & & \\
\hline \multirow[t]{2}{*}{$\mathrm{UE}+\mathrm{MRI}$} & + & 69 & 1 & 70 & 47.314 & 0.630 & 0.001 \\
\hline & - & 0 & 2 & 2 & & & \\
\hline
\end{tabular}

UE, ultrasound elastography; MRI, magnetic resonance imaging. The P-values refer to Kappa coefficients.

\section{Discussion}

UE, as a novel ultrasound imaging technique based on the measurement of the relative hardness of a lesion against the adjacent normal tissues, has the ability to yield a more accurate estimation compared with conventional ultrasound examination technology in the differentiation of malignant from benign breast lesions (16). A previous study has demonstrated that UE was helpful in the differentiation of benign and malignant breast lesions since the hardness of malignant breast cancer was 2-3 times higher than that of benign cancer (17). MRI is another noninvasive diagnostic tool that is useful in distinguishing soft tissue from other tissues. Therefore, the combination of UE and MRI may be more beneficial to identify the likelihood of benign or malignant breast lesions based on the hardness of the lesions $(18,19)$. As a novel and effective imaging tool, the combination of UE and MRI methods may significantly improve diagnostic accuracy and be more objective.

A previous study showed that the accuracy rate of UE was higher compared with color flow Doppler in the diagnosis of breast cancer (20). In the present study, when the diagnostic methods of UE and MRI were used to distinguish between benign and malignant breast tumor properties, the detection rates were found to be $85.8,64.8$ and $92.7 \%$ in the UE, MRI and UE+MRI groups, respectively. These findings revealed that UE and MRI were effective tools for identifying benign or malignant breast cancer, while the combination of UE and MRI was superior to the single application of UE or MRI.

The ROC curve is an effective method for evaluating the performance of diagnostic tests. In addition, AUC is a common index summarizing the information contained in the curve and reflecting the reliability of diagnostic methods. In the current study, the AUC values were 86.7, 79.2 and 91.4\% in the UE, MRI and UE+MRI methods, respectively, for the diagnosis of breast lesions. The corresponding cut off points for these three methods were defined as 2.81, 3.76 and 3.42, respectively. These cut off points yielded the highest sensitivities of 83.3, 77.7 and $95.8 \%$ for UE, MRI and UE+MRI, respectively. Previous studies reported similar results, observing that the sensitivity of UE ranged between 87.1 and $95.0 \%$ in the diagnosis of breast cancer $(19,21)$. Furthermore, the present study revealed that the accuracy rates of differential diagnosis were 82.5, 75.5 and $95.3 \%$ for UE, MRI and UE+MRI, respectively. Therefore, the diagnostic accuracy rate of the UE+MRI method was significantly higher compared with the single application of UE or MRI. In addition, the three methods had high diagnostic agreement with the pathological diagnosis; however, the diagnostic consistency of UE+MRI was higher compared with the application of UE or MRI alone for the diagnosis of benign and malignant breast masses. Another study demonstrated the same trend, but with higher AUC values of UE's diagnosis on breast mass (22). This difference may be due to a limited number of samples, selection bias and inconsistent sampling of the present study.

In conclusion, UE and MRI are effective tools in the diagnosis of breast cancer, while the combination of UE (SR-based) and MRI is superior to the single use of UE or MRI and can greatly improve the detection rate of malignant breast tumors. These results may be beneficial for the optimized use of UE and MRI in clinical practice, although further studies are warranted to confirm these findings. 


\section{Acknowledgements}

The authors wish to thank Professor Huawen Zhang (Department of Radiology, Nuclear Industry 215 Hospital of Shanxi Province, Xianyang, China) for the technical support concerning UE and MRI.

\section{References}

1. Zheng Q, Wang JF, Dai ZQ, Hu J and Xu Y: Diagnostic value of color doppler ultrasonography, ultrasonic elastograqhy and mammography in the differentiation of breast lesions. J Community Med 11: 14-16, 2013 (In Chinese)

2. Gong NM and Wu J: The Value analysis and evaluation of randomized control of color doppler ultrasound combined CT applying to analyze breast cancer. Chin J CT MRI 131: 51-53, 2015 (In Chinese).

3. Meng SP, Zhang ZP and Wang P: Research on application value of CT, ultrasound and X-ray mammography in the diagnosis of breast carcinoma. Chin J CT MRI 16: 33-35, 2014 (In Chinese).

4. Jiang PL, Wang SH, Jiang DM, Tang LL and YU LL: Study on cancer-related fatigue and disease characteristics of breast cancer patients. China J Mod Med 21: 4443-4449, 2011.

5. Wind JJ and Ammerman JM: Pathologic cervical burst fracture presenting with airway compromise. South Med J 103: 551-553, 2010.

6. Warner E and Causer PA: MRI surveillance for hereditary breast-cancer risk. Lancet 365: 1747-1749, 2005.

7. Wu H and Ouyang QC: Protective effect of dexrazoxane on anthracyclines cardio toxicity of the female breast cancer patients with postoperative chemotherapy. China J Mod Med 20: 2188-2194, 2010

8. Zheng Y, Wu CX and Wu F: Status and trends of breast cancer mortality in Chinese females. Zhonghua Yu Fang Yi Xue Za Zhi 45: 150-154, 2011 (In Chinese).

9. Xu JX: Application of ultrasound elastography in qualitative diagnosis of solid breast masses. China Health Ind 8: 147-148, 2014 (In Chinese).

10. Zhong XF: The investigation of the diagnostic value of ultrasound elastography in breast masses. Contemporary Med 20: $149-150,2014$.
11. Liberman L, Morris EA, Dershaw DD, Abramson AF and Tan LK: MR imaging of the ipsilateral breast in women with percutaneously proven breast cancer. AJR Am J Roentgenol 180: 901-910, 2003.

12. Adamietz BR, Meier-Meitinger M, Fasching P, Beckmann M, Hartmann A, Uder M, Häberle L, Schulz-Wendtland R and Schwab SA: New diagnostic criteria in real-time elastography for the assessment of breast lesions. Ultraschall Med 32: 67-73, 2011.

13. Zhao QL, Ruan LT, Zhang H, Yin YM and Duan SX: Diagnosis of solid breast lesions by elastography 5-point score and strain ratio method. Eur J Radiol 81: 3245-3249, 2012.

14. Gu YJ, Wu B, Zhang S and Yang T: The application experiences of breast imaging reporting and data system in mammographic diagnoses of breast lesion with symptoms. Chin J Radiol 38: 931-936, 2004 (In Chinese).

15. Gu Y, Wu B, Zhang S and Yang T: The application experiences of breast imaging reporting and data system in mammographic diagnoses of breast lesion with symptoms. Chin J Radiol 9: 931-936, 2004 (In Chinese).

16. Hooley RJ, Scoutt LM and Philpotts LE: Breast ultrasonography: State of the art. Radiology 268: 642-659, 2013.

17. Krouskop TA, Wheeler TM, Kallel F, Garra BS and Hall T: Elastic moduli of breast and prostate tissues under compression. Ultrason Imaging 20: 260-274, 1998.

18. Zhi H, Xiao XY and Yang HY: Primary comparison of the diagnostic value of strain ratio measure method and 5-scoring system in ultrasonic elestography for differentiation breast benign and malignant solid lesions with ultrasonic elastography. Chin J Ultrasonography 19: 142-144, 2010 (In Chinese).

19. Zhou J, Zhan W, Dong Y, Yang Z and Zhou C: Stiffness of the surrounding tissue of breast lesions evaluated by ultrasound elastography. Eur Radiol 24: 1659-1667, 2014.

20. Zhang ZM, Zhao L, Wang YL, Liu Y, Wang SL and He Y: Diagnostic value of ultrasonic elastography, color doppler flow imaging and mammography in breast diseases. Chongqing Med 12: 3604-3606, 2013.

21. Wu YQ, Jin M, He LL and Huang AQ: Value of ultrasonic elastography ratios for differentiating malignant and benign breast lesions. Diagnostic Imaging Interventional Radiol 24: 134-137, 2015.

22. Zhi H, Xiao XY, Yang HY, Wen YL, Luo BM and Liang BL: Diagnostic value of strain ratio for differentiation breast benign and malignant solid lesions with ultrasonic elastography. Chin J Ultrasonography 18: 589-591, 2009. 